\title{
UMA DEMONSTRAÇÃO SIMPLES E VISUAL DO EFEITO DO AQUECIMENTO COM MICROONDAS EM REAÇÕES DE POLIADIÇÃO
}

\author{
Bianca L. Sadicoff, Marcia C.V. Amorim* \\ Departamento de Química Orgânica - Instituto de Química - Universidade do Estado do Rio de Janeiro - Pavilhão Haroldo Lisboa \\ da Cunha - 20550-013 - Rio de Janeiro - RJ \\ Marcio C. S. de Mattos* \\ Departamento de Química Orgânica - Instituto de Química - Universidade Federal do Rio de Janeiro - CP 68545 - $21945-970$ - \\ Rio de Janeiro - RJ \\ Recebido em 1/3/99; aceito em 19/10/99

\begin{abstract}
A SIMPLE AND VISUAL DEMONSTRATION OF THE HEATING EFFECT OF MICROWAVES IN POLYADDITION REACTIONS. Polyaddition of commercial monomers is easily performed on a domestic microwave oven. The rate of polymerization depends on the structure of the monomer, power and time of irradiation. This methodology can easily be used to demonstrate the acceleration of organic reactions promoted by microwaves.
\end{abstract}

Keywords: polyaddition; microwave; domestic oven.

\section{INTRODUÇÃO}

Analisando-se o espectro eletromagnético, a região das microondas é a faixa de comprimento de onda que vai de $1 \mathrm{~mm}$ a $1 \mathrm{~m}$ e freqüências de $30 \mathrm{GHz}$ a $300 \mathrm{MHz}^{1-3}$, estando assim entre a radiação do infravermelho e as freqüências de rádio e tendo a vantagem de possuir algumas das propriedades de ondas curtas e outras de ondas longas. Desta forma, as microondas podem ser irradiadas, refletidas e também utilizadas para o aquecimento (segundo A. Zlotorzynski ${ }^{1}$, o aquecimento por meio da energia de microondas é, fundamentalmente, a primeira técnica de aquecimento depois da descoberta do fogo). Este aquecimento se deve à habilidade de certas substâncias de transformarem energia eletromagnética em calor, sendo bem diferente dos processos de aquecimento usuais (condução, irradiação e convecção) $)^{1,4}$.

Estão descritos na literatura vários modelos de reatores de microondas ${ }^{5,6}$. Em um forno de microondas caseiro, as ondas são geradas em um magnetron (um aparelho a vácuo que converte energia elétrica alternada em microonda), guiadas para a cavidade do forno e refletidas para as paredes deste ${ }^{7,8}$. Assim, as substâncias que se encontram no interior do forno absorvem esta energia em diversos pontos e as transferem para o resto do corpo. Consequientemente, ao contrário do aquecimento convencional, onde uma substância é aquecida de fora para dentro, no interior de um forno de microondas o que ocorre é uma transferência de calor dos diversos pontos que absorveram a energia de dentro do corpo para a parte externa do mesmo.

Vários trabalhos vêm sendo publicados sobre aplicações de energia de microondas em química ${ }^{1,3,8}$ e na adaptação de fornos domésticos para este fim ${ }^{2,9,10}$. Todavia, recentemente, diversas reações orgânicas têm sido realizadas com segurança em fornos de microondas domésticos sem qualquer tipo de adaptação $^{11,12}$, sendo, inclusive, uma tecnologia promissora em nível industrial ${ }^{13}$. Nestes casos, observa-se que várias reações "clássicas" podem ser bastante aceleradas (muitas vezes por um fator de $10^{3}$ ) quando o aquecimento é realizado por meio de um forno de microondas caseiro ao invés do aquecimento convencional (placa de aquecimento, bico de Bunsen, etc). Além disso, os rendimentos de reações realizadas em presença

e-mail: amorim@uerj.br

e-mail: mmattos@iq.ufrj.br de microondas costumam ser maiores, há menor formação de subprodutos e não ocorrem decomposições de materiais ${ }^{7,10,13-16}$.

Os polímeros são compostos de peso molecular da ordem de $10^{3}$ a $10^{6}$ (sendo por esse motivo geralmente sólidos) e com estruturas onde se encontram unidades simples ("meros") repetidas ${ }^{17}$. São também materiais de grande interesse industrial e comercial com diferentes aplicações ${ }^{17,18}$, como mostrado na Tabela 1.

Apesar da grande aceleração na velocidade de reações orgânicas ser promovida pelas microondas, este efeito freqüentemente não é visualizado facilmente, visto que ele só é percebido após o isolamento e a identificação dos produtos de reação ${ }^{19}$. Assim, neste trabalho, é relatado um experimento fácil e rápido que permite a visualização da eficiência da irradiação de microondas na polimerização por meio de reação em cadeia pela técnica em massa (isto é, em ausência de solvente) de monômeros de interesse industrial ${ }^{17}$.

\section{METODOLOGIA}

Utilizou-se um forno de microondas caseiro Samsung modelo MW 5630T de prato giratório com $2450 \mathrm{MHz}$ de freqüência e controles digitais de tempo e potência.

A escolha do recipiente utilizado nas reações de polimerização foi o resultado de testes com um recipiente cilíndrico de teflon de $6,0 \mathrm{~cm} \times 3,5 \mathrm{~cm}$ (d) com um orifício central de 5,0 $\mathrm{cm} \times 2,0 \mathrm{~cm}$ ou um tubo de ensaio de pyrex de $15,0 \mathrm{~cm} \times 1,5$ $\mathrm{cm}$ (d). Nestes testes observou-se a variação da temperatura de diferentes solventes quando irradiados com energia de microondas em determinado tempo ${ }^{20}$.

Os monômeros estudados na polimerização foram estireno, acetato de vinila e metacrilato de metila, sendo $10 \mathrm{~mL}$ de cada um colocados no orifício central do recipiente cilíndrico de teflon e, quando necessário, adicionou-se $50 \mathrm{mg}$ de AIBN (azobisisobutironitrila). A potência de irradiação foi determinada pela metodologia de Watkins ${ }^{7}$.

Os polímeros assim obtidos foram purificados por solubilização-precipitação e caracterizados por espectroscopia na região de infravermelho com transformada de Fourier num aparelho Perkin-Elmer 1600 FT-IR. As massas molares ponderais médias $\left(\mathrm{M}_{\mathrm{w}}\right)$ dos polímeros foram determinadas por cromatografia de permeação em gel utilizando um cromatógrafo Waters 410 e poliestireno na faixa de $10^{3}-10^{6}$ como padrão e THF como solvente. 
Tabela 1. Polímeros de interesse industrial.

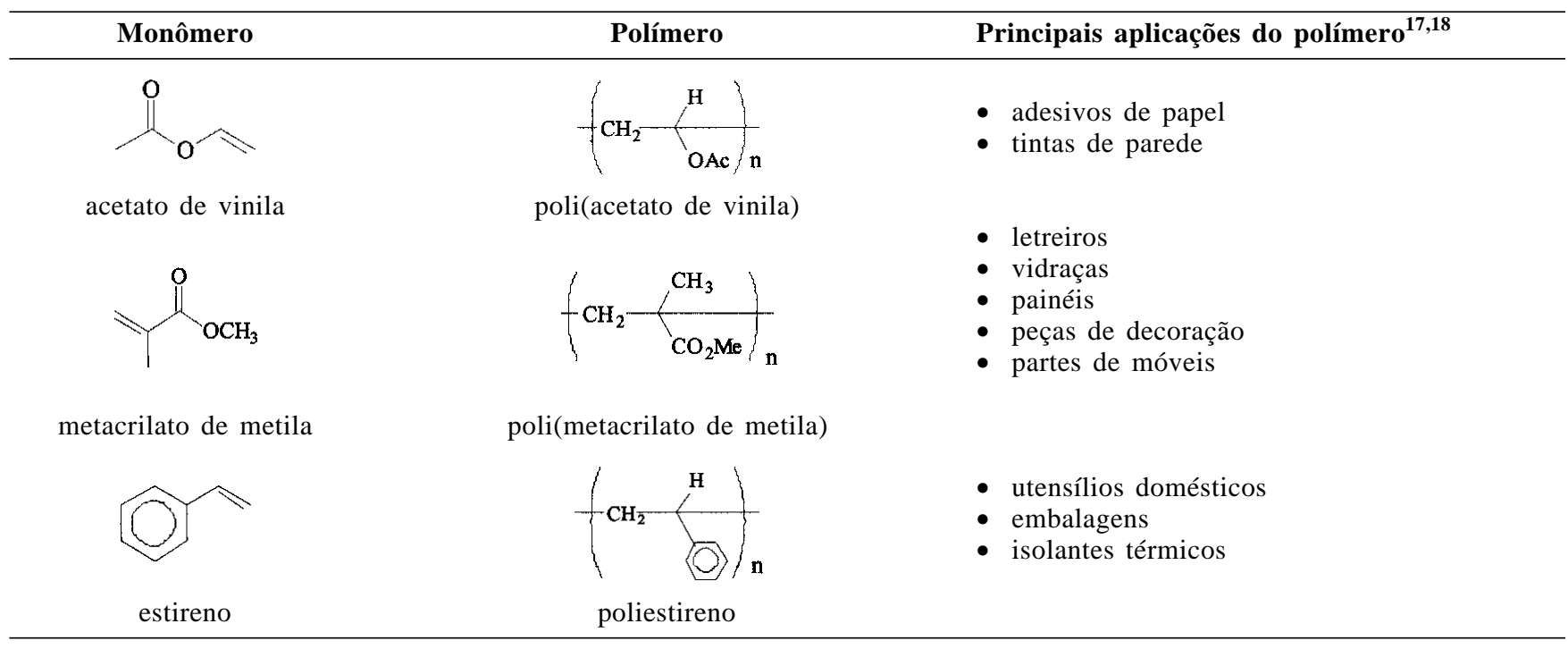

poliestireno: purificado por solubilização em acetato de etila e precipitação em etanol. $I V:$ v 3020, 2940, 1594, 1480, 1432, $750,692,540 \mathrm{~cm}^{-1}$.

poli(acetato de vinila): purificado por solubilização em acetona e precipitação em tetracloreto de carbono. $I V: \vee 2970,2930$, $1428,1258,693,615 \mathrm{~cm}^{-1}$.

poli(metacrilato de metila): purificado por solubilização em acetona e precipitação em etanol. $I V: \vee 2970,1724,1450,1236$, $1150,970,746 \mathrm{~cm}^{-1}$.

\section{RESULTADOS E DISCUSSÃO}

No teste realizado para a escolha do recipiente a ser utilizado nas reações de polimerização com irradiação de microondas, foram obtidas as curvas representadas na Fig. 1. Essa figura mostra a variação de temperatura na irradiação de tetracloreto de carbono e hexano em recipientes de pyrex e teflon ${ }^{20}$. Não foi observada uma variação significativa de temperatura quando os solventes foram irradiados no recipiente de teflon. Todavia, quando a irradiação foi feita no recipiente de pyrex, foi observado um aumento considerável da temperatura com o tempo de irradiação. A perda dielétrica (eficiência de conversão de radiação eletromagnética em calor $^{1}$ ) do vidro ${ }^{21}$ é $1,7 \times 10^{-2}$, enquanto que a do teflon ${ }^{22}$ é $3,0 \times$ $10^{-4}$, indicando que o teflon se aquece bem menos quando irradiado pelas microondas. Como é sabido que tanto o tetracloreto de carbono quanto o hexano praticamente não absorvem a energia das microondas ${ }^{7}$, o aumento da temperatura observado nestes solventes, quando irradiados no recipiente de pyrex, pode ser interpretado como transferência de calor do pyrex para o solvente.

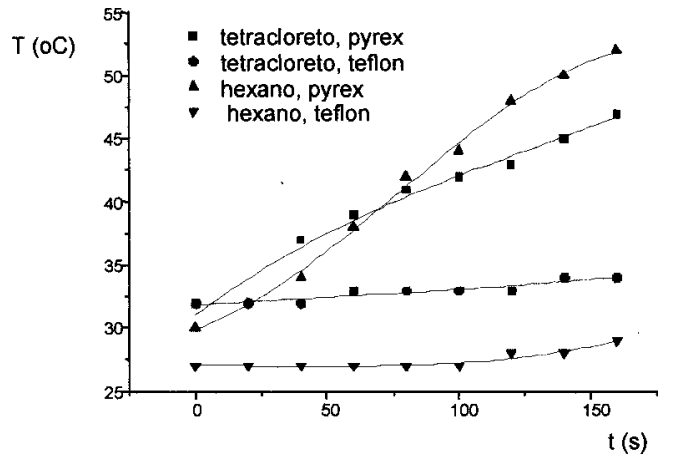

Figura 1. Temperatura do hexano e do tetracloreto de carbono em função do tempo de irradiação de microondas $(295 \mathrm{~W})$ em recipientes de pyrex e de teflon.
De posse desses resultados, os monômeros estireno, acetato de vinila e metacrilato de metila foram irradiados no recipiente de teflon, visto que, a transferência de calor entre ele e o seu conteúdo, caso ocorra, deve ser muito pequena, garantindo assim apenas a absorção da energia das microondas pelo monômero. Na Tabela 2 estão apresentadas as condições reacionais e os aspectos dos polímeros obtidos.

A irradiação do estireno com baixas potências $(<500 \mathrm{~W})$ de microondas e por tempos prolongados (>30 min) levou à formação de um líquido viscoso, indicativo de sua baixa conversão no polímero ou então de formação de oligômeros. Todavia, a irradiação por 20 min a $687 \mathrm{~W}$ levou à formação de um sólido maleável de massa molar média da ordem de $9.400 \mathrm{~g} / \mathrm{mol}$, indicativo de uma polimerização mais completa. Esse resultado indicava que a polimerização do estireno promovida por microondas era possível, porém o polímero obtido não era um sólido rígido, característica do poliestireno ${ }^{17}$. Assim, adicionou-se $0,05 \%$ (em peso) de AIBN (iniciador de polimerização) e através da irradiação a $193 \mathrm{~W}$ por apenas $10 \mathrm{~min}$, obteve-se um sólido rígido de massa molar média da ordem de $48.400 \mathrm{~g} / \mathrm{mol}$.

De posse desses resultados, as polimerizações do acetato de vinila e do metacrilato de metila foram efetuadas pela irradiação dos respectivos monômeros em presença de AIBN, o que levou aos polímeros como sólidos rígidos. Foi observado que nestes dois casos a polimerização foi completa em um tempo bem menor do que o empregado para o estireno. Esse fato pode ser explicado pela baixa polaridade do estireno (quando comparada com a do metacrilato de metila e a do acetato de vinila), o que não permite uma absorção tão efetiva da energia de microondas quanto os outros dois monômeros ${ }^{4,20}$

Em todos os casos estudados, os pesos moleculares foram elevados e a conversão foi superior a $90 \%$, com exceção do acetato de vinila devido à perda por volatilidade. Também foi observado que os polímeros foram obtidos com tempos de reação em média 1/60 dos utilizados em reações sob aquecimento convencional ${ }^{17}$.

A polimerização por meio de reação em cadeia, uma vez iniciada, produz uma cadeia polimérica cujo crescimento rápido resulta na formação de moléculas de alto peso molecular logo no início da reação ${ }^{17}$. Como o andamento da reação de poliadição em massa gera um aumento progressivo na viscosidade do meio, o aspecto do material final pode ser indicativo da conversão do monômero no polímero e da visualização da ocorrência da reação. Assim, essa metodologia pode ser facilmente empregada em aulas de química experimental para demonstrar o efeito da aceleração de reações orgânicas promovidas pelas microondas. 
Tabela 2. Condições reacionais e características dos polímeros obtidos.

\begin{tabular}{|c|c|c|c|c|c|c|}
\hline Monômero & $\begin{array}{c}\mathrm{t} \\
(\min )\end{array}$ & $\begin{array}{l}\text { potência } \\
\text { (W) }\end{array}$ & iniciador & $\begin{array}{c}\text { conversão } \\
(\%)\end{array}$ & $\begin{array}{c}\mathrm{M}_{\mathrm{W}}{ }^{a} \\
(\mathrm{~g} / \mathrm{mol})\end{array}$ & características do polímero \\
\hline estireno & $>30$ & $<500$ & - & - & - & líquido viscoso \\
\hline estireno & 20 & 687 & - & ca. 100 & 9.370 & sólido maleável \\
\hline estireno & 10 & 163 & AIBN & 97 & 48.423 & sólido rígido e transparente \\
\hline acetato de vinila & 5 & 163 & AIBN & 90 & 150.210 & sólido rígido e transparente \\
\hline metacrilato de metila & 10 & 163 & AIBN & 65 & 176.700 & sólido rígido e transparente \\
\hline
\end{tabular}

${ }^{a}$ massa molar ponderal média determinada por cromatografia de permeação em gel.

\section{CONCLUSÕES}

A partir dos resultados obtidos pode-se verificar a influência do tempo, potência e estrutura dos monômeros na absorção da energia de microondas. Como são produzidos polímeros sólidos partindo-se de monômeros líquidos, esta pode ser uma metodologia simples e fácil de se demonstrar a influência de microondas na aceleração de reações orgânicas.

\section{AGRADECIMENTOS}

Agradecemos a leitura, críticas e sugestões de Antonio Manzolillo Sanseverino e ao Instituto de Macromoléculas Prof ${ }^{\mathbf{a}}$ Eloisa Mano (IMA/UFRJ) pelas análises dos polímeros. B.L.S. agradece a bolsa de estudos concedida pelo CETREINA/UERJ.

\section{REFERÊNCIAS}

1. Zlotorzynski, A.; Critical Rev. Anal. Chem. 1995, 25, 43.

2. Mingos, D. M. P.; Baghurst, D. R.; Chem. Soc. Rev. 1991, $20,1$.

3. Galena, S. A.; Chem. Soc. Rev. 1997, 26, 233.

4. Gabriel, C.; Gabriel, S.; Grant, E. H.; Halstead, B. S. J.; Mingos, D. M. P.; Chem. Soc. Rev. 1998, 27, 213.

5. Strauss, C. R.; Trainor, R. W.; Aust. J. Chem. 1995, 48, 1665.

6. Loupy, A.; Petit, A.; Hamelin, J.; Texier-Boullet, F.; Jacquault, P.; Mathé, D.; Synthesis 1998, 1213.

7. Watkins, K. W.; J. Chem. Educ. 1983, 60, 1043.
8. Abramovitch, R.; Org. Prep. Proc. Int. 1991, 23, 683.

9. Pecoraro, E.; Davolos, M. R.; Jafelicci Jr, M.; Quim. Nova 1997, 20, 89.

10. Caddick, S.; Tetrahedron 1995, 51, 10403.

11. Bose, A. K.; Banik, B. K.; Barakat, K. J.; Manhas, M. S.; Synlett 1993, 575.

12. Bose, A. K.; Banik, B. K.; Lavlinskais, N.; Jayaraman, M.; Manhas, M. S.; CHEMTEC 1997, 27 (setembro), 18.

13. Stinson, S.; Chem. Eng. News 1996, 74 (20 de Maio), 45.

14. Sampath Kumar, H. M.; Subbareddy, B. V.; Anjaneyulu, S.; Yadav, J. S., Synth. Commun. 1998, 28, 3811.

15. Parquet, E.; Lin, Q.; J. Chem. Educ. 1997, 74, 1225.

16. Alajarín, R.; Vaquero, J. J.; García Navio, J. L.; AlvarezBuilla, J.; Synlett 1992, 297.

17. Mano, E. B.; Introdução a Polímeros; Edgar Blücher; São Paulo, 1985

18. Mark, H. F.; Bikales, N. M.; Overberger, C. G.; Menges, G. (Ed); Encyclopedia of Polymer Science and Engineering, 2 $2^{\text {a }}$ ed.; John Wiley and Sons; Nova York, 1990.

19. Bari, S. S.; Bose, A. K.; Chaudhary, A. G.; Manhas, M. S.; Raju, V. S.; Robb, E. W.; J. Chem. Educ. 1992, 69, 938.

20. Sadicoff, B. L.; Amorim, M. C. V.; de Mattos, M. C. S., II Encontro Técnico de Materiais e Química - Anais; Rio de Janeiro, 1997, p. 153.

21. Weast, R. C. (Ed); Handbook of Chemistry and Physics, 68 a ed.; CRC; Boca Raton, 1987 - 1988.

22. Jordan, C.; Galy, J.; Pascault, J.-P.; Moré, C.; Delmotte, M.; Jullien, H.; Polymer Eng. Sci. 1995, 35, 233. 